TRANSACTIONS OF THE

AMERICAN MATHEMATICAL SOCIETY

Volume 353, Number 1, Pages 411-426

S 0002-9947(00)02590-3

Article electronically published on September 13, 2000

\title{
OPTIMAL FILTRATIONS ON REPRESENTATIONS OF FINITE DIMENSIONAL ALGEBRAS
}

\author{
LIEVEN LE BRUYN
}

\begin{abstract}
We present a representation theoretic description of the non-empty strata in the Hesselink stratification of the nullcone of representations of quivers. We use this stratification to define optimal filtrations on representations of finite dimensional algebras. As an application we investigate the isomorphism problem for uniserial representations.
\end{abstract}

\section{Optimal Filtrations}

1.1. Let $A$ be a finite dimensional algebra over an algebraically closed field $k$. In this paper we want to parameterize isomorphism classes of finite dimensional $A$ modules having a specific Jordan-Hölder sequence. In particular, we want to relate the recent results of K. Bongartz and B. Huisgen-Zimmermann [3, 4, 1] on uniserial modules to the Hesselink stratification of nullcones.

By Morita theory we may reduce to the case that $A$ is a basic algebra. That is, all simple $A$-modules are one dimensional. In this case we can write $A$ as the quotient of the path algebra of a quiver and relate finite dimensional $A$-modules to representations of this quiver.

1.2. A quiver $Q$ is a directed graph on a finite set of vertices $\left\{v_{1}, \ldots, v_{n}\right\}$. Let $a_{i j}$ be the number of directed arrows from $v_{i}$ to $v_{j}$ (or loops if $v_{i}=v_{j}$ ). The Euler-form of $Q$ is the bilinear form

$$
\chi: \mathbb{Z}^{n} \times \mathbb{Z}^{n} \rightarrow \mathbb{Z}
$$

determined by the matrix $\chi=\left(\chi_{i j}\right)_{i, j} \in M_{n}(\mathbb{Z})$ with entries

$$
\chi_{i j}=\delta_{i j}-a_{i j}
$$

Clearly, $\chi$ encodes the structure of the directed graph $Q$.

A representation $V$ of a quiver $Q$ of dimension vector $\alpha=\left(a_{1}, \ldots, a_{n}\right) \in \mathbb{N}^{n}$ assigns to every arrow $v_{i} \stackrel{\phi}{\longrightarrow} v_{j}$ in $Q$ a matrix $V(\phi) \in M_{a_{j} \times a_{i}}(k)$. The set of all $\alpha$-dimensional representations form an affine space,

$$
\operatorname{Rep}(Q, \alpha)=\bigoplus_{i, j=1}^{n} M_{a_{j} \times a_{i}}(k)^{\oplus a_{i j}}
$$

where $a_{i j}$ is the number of arrows in $Q$ from $v_{i}$ to $v_{j}$.

Received by the editors May 26, 1998 and, in revised form, April 30, 1999.

2000 Mathematics Subject Classification. Primary 16G20, 16 R30.

The author is a research director at the FWO (Belgium). 
There is a natural action of the basechange group $G L(\alpha)=G L_{a_{1}} \times \ldots \times G L_{a_{n}}$ on $\operatorname{Rep}(Q, \alpha)$, the orbits correspond to isomorphism classes of representations.

The path algebra $k Q$ of the quiver $Q$ is a vectorspace with basis the oriented paths in $Q$ of length $\geq 0$ and the multiplication induced by concatenation of paths. If $p$ and $q$ are paths in $Q$, we denote by $q p$ the path obtained by concatenating $q$ after $p$.

Representations of $Q$ can be viewed as finite dimensional representations of $k Q$. In this way, representations form an Abelian category and one defines homomorphisms, extensions, etc., in the obvious way. If $V \in \operatorname{Rep}(Q, \alpha)$ and $W \in \operatorname{Rep}(Q, \beta)$, then

$$
\chi(\alpha, \beta)=\operatorname{dim}_{k} \operatorname{Hom}(V, W)-\operatorname{dim}_{k} \operatorname{Ext}^{1}(V, W)
$$

1.3. We fix $Q$ to be the quiver corresponding to the basic algebra $A$. That is, if $\left\{S_{1}, \ldots, S_{n}\right\}$ is the set of isoclasses of simple (one-dimensional) $A$-modules, then $Q$ is a quiver on $n$ vertices $\left\{v_{1}, \ldots, v_{n}\right\}$ such that

$$
a_{i j}=\operatorname{dim}_{k} \operatorname{Ext}_{A}^{1}\left(S_{i}, S_{j}\right)
$$

Alternatively, let $\left\{e_{1}, \ldots, e_{n}\right\}$ be a complete set of primitive idempotents of $A$; then the arrows from $v_{i}$ to $v_{j}$ in $Q$ form a $k$-basis of the vectorspace

$$
e_{j} J e_{i} / e_{j} J^{2} e_{i}
$$

where $J$ is the Jacobson radical of $A$. Given a choice of primitive idempotents and bases of these vectorspaces, we can identify $A$ with $k Q / I$ where $I$ is an admissible ideal of $k Q$, that is generated by linear combinations of paths of length $\geq 2$ in the quiver $Q$. Such a choice of idempotents and bases is called a coordinatization of $A$. We will fix a coordinatization, and from now on we identify $A$ with $k Q / I$ and the vertex $v_{i}$ of $Q$ with the primitive idempotent $e_{i}$ of $A$.

To a finite dimensional (left) representation $M$ of $A$ we associate its dimension vector $\alpha=\left(a_{1}, \ldots, a_{n}\right)$, where

$$
a_{i}=\operatorname{dim}_{k} e_{i} \cdot M
$$

The set of all $\alpha$-dimensional representations of $A$ forms an affine algebraic variety $\operatorname{Rep}(A, \alpha)$ which is a closed subvariety of $\operatorname{Rep}(Q, \alpha)$. Recall that a representation $V \in \operatorname{Rep}(Q, \alpha)$ is determined by matrices $V(\phi)$ assigned to every arrow $\phi$ in $Q$. Any element of $k Q_{+}$that is the subspace generated by paths of length $\geq 1$ in $Q$ can be evaluated using these matrices. The closed subvariety $\operatorname{Rep}(A, \alpha)$ is then determined as those $\alpha$-dimensional representations of $Q$ such that every $f \in I$ evaluates to the zero matrix.

Clearly, $\operatorname{Rep}(A, \alpha)$ is a $G L(\alpha)$-subvariety of $\operatorname{Rep}(Q, \alpha)$ and orbits correspond to isomorphism classes of $A$-representations.

1.4. We claim that $\operatorname{Rep}(A, \alpha)$ is a subvariety of the nullcone of $\operatorname{Rep}(Q, \alpha)$ under the action of $G L(\alpha)$. By definition, this nullcone is the subvariety of $V \in \operatorname{Rep}(Q, \alpha)$ such that the Zariski orbit closure $\overline{G L(\alpha) . V}$ contains the zero representation.

Consider the one-dimensional simple $A$-representation

$$
S_{i}=e_{i} A / e_{i} J=e_{i} k Q / e_{i} k Q_{+}
$$

Then the zero representation of $\operatorname{Rep}(Q, \alpha)$ is contained in $\operatorname{Rep}(A, \alpha)$ and corresponds to the $\alpha$-dimensional semisimple $A$-module

$$
S(\alpha)=S_{1}^{\oplus a_{1}} \oplus \ldots \oplus S_{n}^{\oplus a_{n}}
$$


Let $M \in \operatorname{Rep}(A, \alpha)$ and consider a Jordan-Hölder sequence

$$
0=M_{0} \subset M_{1} \subset M_{2} \subset \ldots \subset M_{a}=M
$$

with $M_{i} / M_{i-1}$ a simple (hence one dimensional) $A$-module. Therefore, $a=\sum_{i} a_{i}$, and $M^{s s}=\bigoplus_{i} M_{i} / M_{i-1}$ is the semisimple $A$-module $S(\alpha)$.

Take a $k$-vectorspace description $M=\bigoplus_{i=1}^{a} k m_{i}$, where $m_{i}$ is a basis vector of $M_{i} / M_{i-1}$. Then there is a one-parameter subgroup $\lambda$ of $G L_{a}$ with respect to this basis;

$$
\lambda(t)=\left[\begin{array}{llll}
t^{d} & & & \\
& t^{d-1} & & \\
& & \ddots & \\
& & & t
\end{array}\right]
$$

lies in $G L(\alpha) \hookrightarrow G L_{a}$ and has the property that

$$
\lim _{t \longrightarrow 0} \lambda(t) \cdot M=M^{s s}=S(\alpha)
$$

whence $M$ is contained in the nullcone.

For this reason we first have to study the nullcone $\operatorname{Null}(Q, \alpha)$ of $\operatorname{Rep}(Q, \alpha)$ under the action of the base-change group $G L(\alpha)$.

1.5. By the Hilbert criterium (see for example [8, III.2.3]) we know that $V \in$ $\operatorname{Rep}(Q, \alpha)$ lies in the nullcone $\operatorname{Null}(Q, \alpha)$ if and only if there is a one-parameter subgroup

$$
\lambda: k^{*} \hookrightarrow G L(\alpha)=G L_{a_{1}} \times \ldots G L_{a_{n}} \hookrightarrow G L_{a}
$$

such that

$$
\lim _{t \longrightarrow 0} \lambda(t) \cdot V=0 \quad \text { in } \operatorname{Rep}(Q, \alpha)
$$

By $T_{a_{i}}$ we denote the standard maximal torus of diagonal matrices in $G L_{a_{i}}$, and with $a=\sum_{i} a_{i}$ the group $T_{a}=\prod_{i} T_{a_{i}}$ is a maximal torus of $G L(\alpha)$. Up to conjugation in $G L(\alpha)$, or equivalently replacing $V$ by another point in its orbit, we may assume that $\lambda$ lies in $T_{a}$ and is of the form

$$
\lambda(t)=\left[\begin{array}{ccc}
t^{\lambda_{1}} & & \\
& \ddots & \\
& & t^{\lambda_{a}}
\end{array}\right] \hookrightarrow\left[\begin{array}{lll}
G L_{a_{1}} & & \\
& \ddots & \\
& & G L_{a_{n}}
\end{array}\right] \hookrightarrow G L_{a}
$$

with the $\lambda_{i} \in \mathbb{Z}$.

Recall that the weights of $T_{a}$ are isomorphic to $\mathbb{Z}^{a}$, having canonical generators $\pi_{i}$ for $1 \leq i \leq a$. Decompose the interval

$$
[1 \ldots a]=\bigcup_{v=1}^{n} I_{v}
$$

into vertex-intervals

$$
I_{v}=\left[\sum_{i=0}^{v-1} a_{i}+1 \ldots \sum_{i=0}^{v} a_{i} .\right]
$$

If we denote $\pi_{i j}=\pi_{j}-\pi_{i}$, we have the weight space decomposition

$$
\operatorname{Rep}(Q, \alpha)=\bigoplus_{\pi_{i j}} \operatorname{Rep}(Q, \alpha)_{\pi_{i j}}
$$


where $\pi_{i j}$ occurs with a non-zero weightspace if and only if $i \in I_{v}, j \in I_{v^{\prime}}$ and in the quiver $Q$ there is an arrow $v \rightarrow v^{\prime}$.

Using this weightspace decomposition, we can write $V=\sum_{\pi_{i j}} V_{i j}$. The condition $\lim _{t \rightarrow 0} \lambda(t) . V=0$ in $\operatorname{Rep}(Q, \alpha)$ with $\lambda$ determined by $\left(\lambda_{1}, \ldots, \lambda_{a}\right) \in \mathbb{Z}^{a}$ is then equivalent to the condition

$$
\lambda_{j}-\lambda_{i} \geq 1 \text { whenever } V_{i j} \neq 0
$$

1.6. Assume $V \in \operatorname{Null}(Q, \alpha)$. Consider the set

$$
E_{V}=\left\{(i, j) \mid V_{i j} \neq 0\right\} .
$$

There exists a unique $a$-tuple $\mu_{V}=\left(\mu_{1}, \ldots, \mu_{a}\right) \in \mathbb{Q}^{a}$ such that $\mu_{j}-\mu_{i} \geq 1$ for all $(i, j) \in E_{V}$ and such that the norm

$$
\left|\mu_{V}\right|=\mu_{1}^{2}+\ldots \mu_{a}^{2}
$$

is minimal.

There is a unique $\lambda_{V}=\left(\lambda_{1}, \ldots, \lambda_{a}\right) \in \mathbb{Z}^{a}$ satisfying

$$
\lambda_{V} \in \mathbb{N} \mu_{V} \quad \text { and } \operatorname{gcd}\left(\lambda_{1}, \ldots, \lambda_{a}\right)=1
$$

The corresponding one-parameter subgroup $\lambda_{V}: k^{*} \hookrightarrow T_{a}$ is called the best oneparameter subgroup for $V$ with respect to the maximal torus $T_{a}$.

We can repeat this procedure for any point $V^{\prime}=g . V$ in the $G L(\alpha)$-orbit of $V$. Assume $V^{\prime}$ is such that $\left|\mu_{V^{\prime}}\right|$ is minimal. We then say that

$$
g^{-1} \lambda_{V^{\prime}} g: k^{*} \hookrightarrow G L(\alpha)
$$

is an optimal one parameter subgroup for $V$ in $G L(\alpha)$. By $\Lambda(V)$ we denote the set of all optimal one-parameter subgroups for $V$ in $G L(\alpha)$.

We recall from [9, Prop. 4.3] and [6] that one-parameter subgroups of $G L(\alpha)$ correspond to filtrations. Let $\lambda: k^{*} \hookrightarrow G L(\alpha)$ be a one-parameter subgroup and take for any vertex $v_{i}$ in $Q$ the decomposition

$$
\mathbb{C}^{a_{i}}=\bigoplus W_{i}^{(m)}
$$

where $\lambda(t)$ acts on the weight space $W_{i}^{(m)}$ as multiplication by $t^{m}$. Consider the filtration

$$
W_{i}^{(\geq m)}=\bigoplus_{m^{\prime} \geq m} W_{i}^{\left(m^{\prime}\right)}
$$

Let $W \in \operatorname{Rep}(Q, \alpha)$; then under the action of $\lambda$ the components of the maps $W(\phi)$ corresponding to an arrow $\phi$ of the quiver

$$
W(\phi)^{\left(m^{\prime}-m\right)}: W_{i}^{(m)} \stackrel{\phi(W)}{\longrightarrow} W_{j}^{\left(m^{\prime}\right)}
$$

are multiplied by $t^{m^{\prime}-m}$. Therefore, $\lim _{t \longrightarrow 0} \lambda(t) . W$ exists if and only if $W(\phi)^{\left(m^{\prime}-m\right)}=$ 0 for all $m^{\prime}<m$. This in turn happens if and only if $W(\phi)$ induces a map $W_{i}^{(\geq m)} \rightarrow W_{j}^{(\geq m)}$ for all $m$; that is, if and only if the subspaces $W_{i}^{(\geq m)}$ determine subrepresentations $W_{(m)}$ of $W$. Thus a one-parameter subgroup $\lambda$ for which $\lim _{t \rightarrow 0} \lambda(t) . W$ exists determines a filtration of $W$

$$
\ldots \supset W_{(m)} \supset W_{(m+1)} \supset \ldots
$$

indexed by $\mathbb{Z}$ and such that $W_{(m)}=W$ for $m$ small. 
Definition 1.1. In particular, let $\lambda_{V}$ be an optimal one-parameter subgroup for $V$ in $G L(\alpha)$. Then $\lim _{t \rightarrow 0} \lambda_{V}(t) . V=0$, and we obtain a decreasing filtration $V_{(m)}$ of $V$ with associated graded representation the zero representation in $\operatorname{Rep}(Q, \alpha)$.

We call this an optimal filtration on $V$. If $V=M \in \operatorname{Rep}(A, \alpha)$, then the subrepresentations $M_{(m)}$ are $A$-representations and we call the corresponding filtration an optimal filtration for the $A$-representation $M$.

1.7. Two representations $V, W \in N u l l(Q, \alpha)$ are said to belong to the same blade if and only if $\Lambda(V)=\Lambda(W)$, where $\Lambda(V)$ denotes as before the set of all optimal 1-parameter subgroups for $V$. By $[V]$ we denote the blade determined by $V$.

The representations $V, W \in \operatorname{Null}(Q, \alpha)$ are said to belong to the same stratum if and only if $\Lambda(V)=\Lambda(g . W)$ for some $g \in G L(\alpha)$. Thus, $G L(\alpha)$. $[V]$ is the stratum determined by $V$.

Let $V \in N u l l(Q, \alpha)$, take $\lambda \in \Lambda(V)$ and define

$$
S(V)=\bigoplus_{\left(\pi_{i j}, \lambda\right) \geq 1} \operatorname{Rep}(Q, \alpha)_{\pi_{i j}}
$$

where $\left(\pi_{i j}, \lambda\right)=\lambda_{j}-\lambda_{i}$. Then, $S(V)$ is a linear subspace of $N u l l(Q, \alpha)$ and by [2, Prop. 4.2] we have that the blade [V] of $V$ is a Zariski open subset of $S(V)$ and the stratum $G L(\alpha)$. $[V]$ is a Zariski open subset of the irreducible variety $G L(\alpha) . S(V)$.

Let $P(\lambda)$ be the parabolic subgroup of $G L(\alpha)$ associated with $\lambda \in \Lambda(V)$. Recall that $P(\lambda)$ consists of those $g \in G L(\alpha)$ such that $\lim _{t \longrightarrow 0} \lambda(t) \cdot g \cdot \lambda(t)^{-1}$ exists.

This parabolic subgroup of $G L(\alpha)$ has unipotent radical $U(\lambda)$ consisting of those elements such that the above limit is equal to the unit element and has Levi subgroup $L(\lambda)$ which is a product of $G L_{j}$ 's determined by the multiplicities of the $\lambda_{i}$. For more details we refer to 8 III.2.5].

The parabolic subgroup $P(\lambda)$ acts on $S(V)$ and hence on $G L(\alpha) \times S(V)$ by

$$
p \cdot(g, W)=\left(g p^{-1}, p . W\right)
$$

Further, there is also an action of $P(\lambda)$ on $G L(\alpha) \times \operatorname{Rep}(Q, \alpha)$, and the natural map

$$
G L(\alpha) \times \operatorname{Rep}(Q, \alpha) \rightarrow G L(\alpha) / P(\lambda) \times \operatorname{Null}(Q, \alpha)
$$

sending $(g, W)$ to $(g P(\lambda), g . W)$ is seen to be a geometric quotient for this action. That is, points of $G L(\alpha) / P(\lambda) \times N u l l(Q, \alpha)$ classify the $P(\lambda)$-orbits in $G L(\alpha) \times$ $\operatorname{Rep}(Q, \alpha)$.

We will denote this quotient by $G L(\alpha) \times{ }^{P(\lambda)} \operatorname{Rep}(Q, \alpha)$, which is a vectorbundle over the flag variety $G L(\alpha) / P(\lambda)$ with fiber $\operatorname{Null}(Q, \alpha)$.

By $G L(\alpha) \times{ }^{P(\lambda)} S(V)$ we denote the image in this quotient of $G L(\alpha) \times S(V)$. One verifies that it is a (not necessarily trivial) vectorbundle over the flag variety $G L(\alpha) / P(\lambda)$ with typical fiber $S(V)$.

In particular, it is a smooth variety of dimension $\operatorname{dim} G L(\alpha)-\operatorname{dim} P(\lambda)+$ $\operatorname{dim} S(V)$, and we have natural morphisms

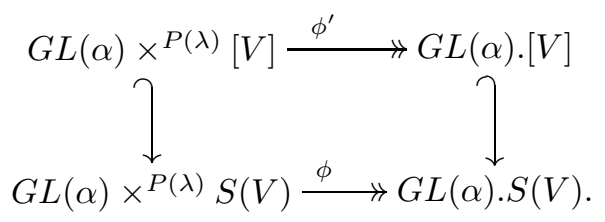


From [2] Th. 4.7] we recall that $\phi$ is birational and a resolution of singularities. Concluding, we obtain the following

Theorem 1.2 (Hesselink). In the above notation:

1. The stratum $G L(\alpha)$. $[V]$ is a smooth irreducible subvariety of $\operatorname{Null}(Q, \alpha)$.

2. The Zariski closure of this stratum is equal to $G L(\alpha) . S(V)$.

3. The desingularization of this closure is a vectorbundle over the flag variety $G L(\alpha) / P(\lambda)$ of rank the dimension of $S(V)$.

4. There is a natural one-to-one correspondence between $G L(\alpha)$-orbits in the stratum $G L(\alpha) \cdot[V]$ and $P(\lambda)$-orbits in the blade $[V] \hookrightarrow S(V)$.

\section{Combinatorics of Strata}

2.1. In order to apply the Hesselink stratification of the nullcone $\operatorname{Null}(Q, \alpha)$ we need to describe the non-empty strata explicitly. In this section we give a representation theoretic solution of this problem.

First, we will compile a finite list $\operatorname{List}(Q, \alpha)$ of $a$-tuples $\mu \in \mathbb{Q}^{a}$ which can determine a stratum $S t_{\mu}$. To each $\mu \in \operatorname{List}(Q, \alpha)$ we will associate a directed quiver $Q_{\mu}$, a dimension vector $\alpha_{\mu}$ and a character $\theta_{\mu}$, and prove that $S t_{\mu} \neq \emptyset$ if and only if there are $\theta_{\mu}$-semistable representations in $\operatorname{Rep}\left(Q_{\mu}, \alpha_{\mu}\right)$ as defined in [6]. Finally, we give a combinatorial solution to this existence problem, using results of A. Schofield [11].

2.2. We will describe the list $\operatorname{List}(Q, \alpha)$. Fix the maximal torus $T_{a}$ of $G L(\alpha)$ and let $\Pi$ be the set of weights of $T_{a}$ having a nontrivial weight space in $\operatorname{Rep}(Q, \alpha)$. Recall that

$$
\Pi=\left\{\pi_{i j}=\pi_{j}-\pi_{i} \mid i \in I_{v}, j \in I_{v^{\prime}} \text { and } \exists v \stackrel{\phi}{\longrightarrow} v^{\prime} \in Q\right\}
$$

A subset $R \subset \Pi$ is said to be unstable if there exists a coweight $\mu=\left(\mu_{1}, \ldots, \mu_{a}\right) \in$ $\mathbb{Q}^{a}$ such that $\mu_{j}-\mu_{i} \geq 1$ for all $\pi_{i j} \in R$.

If $R$ is unstable, there is a unique coweight $\mu(R) \in \mathbb{Q}^{a}$ with this property and such that the norm $|\mu(R)|$ is minimal.

We define the saturation $R^{\text {sat }}$ of $R$ to be the subset

$$
R^{s a t}=\left\{\pi_{i j} \in \Pi \mid\left(\pi_{i j}, \mu(R)\right) \geq 1\right\}
$$

and we call $R$ a saturated subset of $\Pi$ whenever $R=R^{\text {sat }}$.

If $R$ is a saturated subset of $\Pi$, we have a corresponding saturated subspace $X_{R}$ of $\operatorname{Null}(Q, \alpha)$ by taking

$$
X_{R}=\bigoplus_{\pi_{i j} \in R} \operatorname{Rep}(Q, \alpha)_{\pi_{i j}}
$$

By [2, Prop. 5.5] one has a bijective correspondence between the $G L(\alpha)$-conjugacy classes of saturated subspaces of $\operatorname{Null}(Q, \alpha)$ and the conjugacy classes of saturated subsets $R \hookrightarrow \Pi$ under the action of the Weyl-group

$$
S_{\alpha}=S_{a_{1}} \times \ldots \times S_{a_{n}}
$$

of $G L(\alpha)$. This correspondence assigns to $R$ the subspace $X_{R}$, and to a saturated subspace the set of non-zero weights of its elements. Hence, the number of Hesselink strata of $\operatorname{Null}(Q, \alpha)$ is smaller than the number of conjugacy classes of saturated subsets $R \hookrightarrow \Pi$ under the Weyl group. 
Clearly, a saturated subset $R \hookrightarrow \Pi$ is determined by its associated coweight $\mu(R)$. We will now describe the possible occurring coweights, following [2, 6.8].

Consider a coweight $\mu=\left(\mu_{1}, \ldots, \mu_{d}\right) \in \mathbb{Q}^{a}$. Then, we can partition $[1 \ldots a]$ into a disjoint union of segments $I$ determined by the properties that there exist rational numbers $p \leq q$ such that

- $\left\{\mu_{i} \mid i \in I\right\}=\{x \in p+\mathbb{Z} \mid p \leq x \leq q\}$

- $I=\left\{1 \leq i \leq a \mid \mu_{i} \in p+\mathbb{Z}, p-1 \leq \mu_{i} \leq q+1\right\}$

We define a coweight $\mu \in \mathbb{Q}^{a}$ to be balanced if and only if for every segment $I$ of $\mu$ we have

$$
\sum_{i \in I} \mu_{i}=0
$$

We call a coweight $\mu \in \mathbb{Q}^{d}$ dominant if and only if for every vertex $v$ we have for all $i, j \in I_{v}$

$$
i \leq j \Rightarrow \mu_{i} \leq \mu_{j}
$$

Finally, for $\mu \in \mathbb{Q}^{a}$ we denote $R(\mu)=\left\{\pi_{i j} \in \Pi \mid \mu_{j}-\mu_{i} \geq 1\right\}$.

Proposition 2.1. With notations as before:

1. If $R$ is a saturated subset of $\Pi$, then $\mu(R)$ is balanced.

2. If $\mu$ is balanced and for every balanced coweight $\mu^{\prime}$ such that $R(\mu) \stackrel{\neq}{\hookrightarrow} R\left(\mu^{\prime}\right)$ we have $|\mu|<\left|\mu^{\prime}\right|$, then $R(\mu)$ is a saturated subset of $\Pi$.

Proof. (Compare with [2, 6.8].) (1) Let $I$ be a segment for $\mu$ and consider, for $\epsilon \in \mathbb{Q}$

$$
\mu_{\epsilon}=\mu+\left(\delta_{1 I}, \ldots, \delta_{a I}\right) \epsilon
$$

where $\delta_{i I}=1$ if $i \in I$ and zero otherwise. By definition of a segment, there exists $\epsilon_{0}>0$ such that for all $\epsilon$ with absolute value $<\epsilon_{0}$ we have

$$
R(\mu)=R\left(\mu_{\epsilon}\right)
$$

By minimality of $|\mu|$, it follows that $\left|\mu_{\epsilon}\right| \geq|\mu|$ whenever $|\epsilon|<\epsilon_{0}$. But,

$$
\left|\mu_{\epsilon}\right|=|\mu|+2 \epsilon \sum_{i \in I} \mu_{i}+\epsilon^{2} \# I
$$

and so $\mu$ must be balanced. If not, we can take $\epsilon<0$ and contradict the minimality of $\mu$.

Statement (2) follows from (1) and the definitions.

Since we are interested in conjugacy classes under the Weyl group of saturated subsets of $\Pi$, we can restrict attention to dominant balanced coweights.

Denote by $\operatorname{List}(Q, \alpha)$ the finite list of dominant balanced coweights satisfying the condition of the second part of the proposition. Any $\mu \in \operatorname{List}(Q, \alpha)$ determines a (conjugacy class) of a saturated subspace

$$
S_{\mu}=\bigoplus_{\pi_{i j} \in R(\mu)} \operatorname{Rep}(Q, \alpha)_{\pi_{i j}}
$$

of $\operatorname{Null}(Q, \alpha)$.

There remains the problem of determining which of these $S_{\mu}$ is the closure of a stratum $S t_{\mu}$ in the Hesselink stratification of $\operatorname{Null}(Q, \alpha)$. 
2.3. Given $\mu \in \operatorname{List}(Q, \alpha)$, we want to determine whether $S_{\mu}$ is of the form $S(V)$ for some representation $V \in \operatorname{Null}(Q, \alpha)$, and if so we want to determine the Zariskiopen blade $[V] \hookrightarrow S(V)$.

In order to achieve this we use some results of F. Kirwan [7, 12.18-12.26]. Let us fix $\mu \in \operatorname{List}(Q, \alpha)$ and define

$$
\mu_{1}(R)=\left\{\pi_{i j} \in \Pi \mid \mu_{j}-\mu_{i}=1\right\}
$$

and

$$
T_{\mu}=\bigoplus_{\pi_{i j} \in \mu_{1}(R)} \operatorname{Rep}(Q, \alpha)_{\pi_{i j}}
$$

Then, there is a natural projection map with vectorspaces as fibers,

$$
S_{\mu} \stackrel{p r}{\rightarrow} T_{\mu}
$$

Let $\lambda$ be the uniquely determined one-parameter subgroup of $T_{a}$ determined by $\mu$, that is, $\lambda \in \mathbb{N} \mu \cap \mathbb{Z}^{a}$ with $\operatorname{gcd}\left(\lambda_{1}, \ldots, \lambda_{a}\right)=1$. The action of $G L(\alpha)$ on $\operatorname{Rep}(Q, \alpha)$ induces actions of

- $P(\lambda)$ on $S_{\mu}$

- $L(\lambda)$ on $T_{\mu}$

There is a Zariski open (but possibly empty) subset $T_{\mu}^{s s}$ of representations $W \in T_{\mu}$ such that $\lambda \in \Lambda(W)$. Specializing [7, 12.24 \& 12.26] to our setting, we obtain

Proposition 2.2. Let $\mu \in \operatorname{List}(Q, \alpha)$. Then, $S_{\mu}=S(V)$ for some $V \in N u l l(Q, \alpha)$ if and only if $T_{\mu}^{s s} \neq \emptyset$. Moreover, in that case we have

1. $[V]=\left\{W \in S_{\mu} \mid \operatorname{pr}(N) \in T_{\mu}^{s s}\right\}$.

2. $T_{\mu}^{s s}$ is an $L(\lambda)$-stable subset of $T_{\mu}$.

3. The fibers of $[V] \stackrel{p r}{\rightarrow} T_{\mu}^{s s}$ are vectorspaces.

4. $[V]$ is a $P(\lambda)$-stable subset of $S_{\mu}=S(V)$.

2.4. In order to verify the condition $T_{\mu}^{s s} \neq \emptyset$ we give an interpretation of the $L(\lambda)$-action on $T_{\mu}$ in a quiver situation.

Let $\mu \in \operatorname{List}(Q, \alpha)$. Let $J_{1}, \ldots, J_{u}$ be the distinct segments of $\mu$ where

$$
J_{i}=\{\underbrace{p_{i}, \ldots, p_{i}}_{\sum_{v=1}^{n} b_{t j_{0}}^{(i)}}, \underbrace{p_{i}+1, \ldots, p_{i}+1}_{\sum_{v=1}^{n} b_{t j_{1}}^{(i)}}, \ldots, \underbrace{p_{i}+k_{i}, \ldots, p_{i}+k_{i}}_{\sum_{v=1}^{n} b_{t j_{k_{i}}}^{(i)}}\}
$$

where $b_{t j_{k}}^{(i)}$ is the number of entries $a \in I_{v}$ such that $\mu_{a}=p_{i}+k$.

Definition 2.3. A quiver $\Gamma$ is said to be a level quiver if we can partition the set of vertices of $\Gamma$ into disjoint subsets $S_{1}, S_{2}, \ldots, S_{l}$ such that the only arrows in $\Gamma$ are from a vertex from $S_{i}$ to one in $S_{i+1}$ for all $1 \leq i \leq l-1$.

Consider for each segment $J_{i}$ with $1 \leq i \leq u$ the level quiver $Q_{i}$ on $n \times\left(k_{i}+1\right)$ vertices $\left\{(v, j) \mid v\right.$ a vertex in $Q$ and $\left.1 \leq j \leq k_{i}+1\right\}$. In $Q_{i}$ there are as many arrows from $(v, k)$ to $\left(v^{\prime}, k+1\right)$ as there are arrows from $v$ to $v^{\prime}$ in $Q$. For the level quiver $Q_{i}$ we take the dimension vector $\alpha_{i}=\left(b_{t j_{k}}^{(i)}\right)_{t k}$.

The quiver $Q_{\mu}$ will be the disjoint union of the level quivers $Q_{i}$ associated to the different segments $J_{i}$ of $\mu$, where $1 \leq i \leq u$. The dimension vector $\alpha_{\mu}$ for $Q_{\mu}$ will be the vector obtained from the dimension vectors $\alpha_{i}$ for $Q_{i}$. 
Theorem 2.4. With notations as above we have identifications

$$
\begin{gathered}
T_{\mu}=\operatorname{Rep}\left(Q_{\mu}, \alpha_{\mu}\right) \\
L(\lambda)=G L\left(\alpha_{\mu}\right)
\end{gathered}
$$

Moreover, the base-change action of $G L\left(\alpha_{\mu}\right)$ on $\operatorname{Rep}\left(Q_{\mu}, \alpha_{\mu}\right)$ coincides under the identifications with the action of $L(\lambda)$ on $T_{\mu}$.

Proof. This is a straightforward but rather tedious verification. Perhaps it is helpful to consider the special case of the $m$-loop quiver treated in [10].

2.5. Using this identification, we will now give a representation theoretic interpretation of the condition $T_{\mu}^{s s} \neq \emptyset$.

We define the character

$$
\chi_{\mu}: L(\lambda)=G L\left(\alpha_{\mu}\right)=\prod_{i=1}^{u} \prod_{v=1}^{n} \prod_{j=0}^{k_{i}} G L_{b_{v j}^{(i)}} \rightarrow k^{*}
$$

determined by sending a tuple (with obvious notation)

$$
\left(g_{v j}^{(i)}\right)_{i v j} \rightarrow \prod_{i, v, j} \operatorname{det}\left(g_{v j}^{(i)}\right)^{m_{v j}^{(i)}}
$$

where the exponents are determined by

$$
m_{v j}^{(i)}=d \cdot\left(p_{i}+j\right)
$$

where $d$ is the least common multiple of all the numerators of the rational numbers $p_{i}$, where $i$ runs over all the segments of $\mu$, that is, $1 \leq i \leq u$.

Let $G(\mu)$ be the kernel of this character and observe that the group of characters of $G(\mu) \cap T_{a}$ corresponds to those $\chi \in \mathbb{Z}^{a}$ such that $(\chi, \mu)=\sum_{z} \chi_{z} \mu_{z}=0$.

But then, [12] Prop 1] applied to our situation implies

Proposition 2.5. $T_{\mu}^{s s}$ is the set of semi-stable points of $T_{\mu}$ with respect to the action of the group $G(\mu)$. That is, $T_{\mu}^{s s}$ is the open subset of $T_{\mu}$ consisting of points $V$ such that there exists a $\chi_{\mu}$ semi-invariant function $f: T_{\mu} \rightarrow \mathbb{C}$ with $f(V) \neq 0$. This in turn means that for all $g \in L(\lambda)$ we have $g . f=\chi_{\mu}(g)^{k} f$ for some $k \in \mathbb{N}$.

Using the identifications of $T_{\mu}=\operatorname{Rep}\left(Q_{\mu}, \alpha_{\mu}\right)$ and of $L(\lambda)=G L\left(\alpha_{\mu}\right)$, we will give a representation theoretic description of the set $T_{\mu}^{s s}$.

Let $\Gamma$ be a quiver on $s$ vertices; then the Grothendieck group $K_{0} k \Gamma$ of the path algebra is $\mathbb{Z}^{s}$ and the isomorphism assigns to the class of a representation $V$ of $\Gamma$ its dimension vector.

If we have an additive function on the Grothendieck group

$$
\theta: K_{0}(k \Gamma) \rightarrow \mathbb{Z}
$$

we can define, following A. King in [6], in analogy with the terminology of vectorbundles on projective varieties, a representation $V \in \operatorname{Rep}(\Gamma, \beta)$ to be

- $\theta$-semistable if $\theta(V)=0$ and for every subrepresentation $W \hookrightarrow V$ we have $\theta(W) \geq 0$.

- $\theta$-stable if it is $\theta$-semistable and the only subrepresentations $W \hookrightarrow V$ satisfying $\theta(W)=0$ are 0 or $N$. 
Returning to the case of interest, the character $\chi_{\mu}$ describes the additive function on the Grothendieck group

$$
\theta_{\mu}: K_{0}\left(k Q_{\mu}\right) \rightarrow \mathbb{Z}
$$

by sending a class of a representation of $Q_{\mu}$ with dimension vector $\left(a_{v j}^{(i)}\right)$ to

$$
\sum_{i v j} m_{v j}^{(i)} a_{v j}^{(i)}
$$

Applying [6] Prop. 3.1] to our situation, we obtain

Proposition 2.6. $T_{\mu}^{s s}$ is the set of $\theta_{\mu}$-semistable representations in $\operatorname{Rep}\left(Q_{\mu}, \alpha_{\mu}\right)=$ $T_{\mu}$.

Therefore, we need to find a method to determine when $\theta$-semistable representations exist.

2.6. Let $\Gamma$ be a quiver on $s$ vertices and $\theta$ an additive function of the Grothendieck group. The subset of $\theta$-semistable representations of $\operatorname{Rep}(\Gamma, \beta)$ is Zariski open (but possibly empty). Hence it suffices to verify whether representations in a general position of $\operatorname{Rep}(\Gamma, \beta)$ are $\theta$-semistable.

If $\beta, \gamma \in \mathbb{N}^{s}$ we write, following A. Schofield in [11], $\gamma \hookrightarrow \beta$ if and only if representations in general position of $\operatorname{Rep}(\Gamma, \beta)$ contain a subrepresentation of dimension vector $\gamma$.

A. Schofield proved in [11] an inductive procedure to verify this condition in terms of the Euler form $\chi$ of $\Gamma$.

Theorem 2.7 (Schofield).

$$
\gamma \hookrightarrow \beta \quad \text { iff } \quad \underset{\delta \hookrightarrow \gamma}{\operatorname{Max}}-\chi(\delta, \beta-\gamma)=0
$$

This result is the final ingredient in our representation theoretic description of the non-empty Hesselink strata of $\operatorname{Null}(Q, \alpha)$.

Theorem 2.8. Let $\mu \in \operatorname{List}(Q, \alpha)$. The saturated subspace of $\operatorname{Null}(Q, \alpha)$

$$
S_{\mu}=\bigoplus_{\pi_{i j} \in R(\mu)} \operatorname{Rep}(Q, \alpha)_{\pi_{i j}}
$$

is the closure of a stratum in the Hesselink stratification of $\operatorname{Null}(Q, \alpha)$ if and only if for the corresponding

- level quiver $Q_{\mu}$,

- dimension vector $\alpha_{\mu}$ and

- additive function $\theta_{\mu}$

the following condition is satisfied:

$$
\beta \hookrightarrow \alpha_{\mu} \Rightarrow \theta_{\mu}(\beta) \geq 0
$$

Moreover, the condition $\beta \hookrightarrow \alpha_{\mu}$ can be verified in terms of the Euler form of $Q_{\mu}$.

If this condition is satisfied, the stratum $S t_{\mu}$ consists of all $W \in S_{\mu}$ such that under the canonical projection

$$
S_{\mu} \stackrel{p r}{\rightarrow} T_{\mu}=\operatorname{Rep}\left(Q_{\mu}, \alpha_{\mu}\right)
$$

$\operatorname{pr}(W)$ is a $\theta_{\mu}$-semistable representation. 
2.7. We can also give a representation theoretic description of the action of the parabolic subgroup $P(\lambda) \hookrightarrow G L(\alpha)$ on the saturated subspace $S_{\mu}$.

Consider the level quiver $Q_{\mu}$ and denote its vertices by $(v, j)^{(i)}$. Recall that $v$ runs over the vertices of $Q, i$ over the different segments of $\mu$ and $j$ over the length of the $i$-th segment $J_{i}$. Further, $J_{i}$ determines a rational number $p_{i}$.

Define the extended quiver $\tilde{Q}_{\mu}$ as follows. Whenever $\left(v_{1}, j_{1}\right)^{\left(i_{1}\right)}$ and $\left(v_{2}, j_{2}\right)^{\left(i_{2}\right)}$ are two vertices of $Q_{\mu}$ satisfying the condition

$$
p_{i_{2}}+j_{2}-p_{i_{1}}-j_{1} \geq 1
$$

then there are as many arrows in $\tilde{Q}_{\mu}$ from $\left(v_{1}, j_{1}\right)^{\left(i_{1}\right)}$ to $\left(v_{2}, j_{2}\right)^{\left(i_{2}\right)}$ as there are arrows in $Q$ from $v_{1}$ to $v_{2}$.

Observe that $Q_{\mu}$ is a subquiver of $\tilde{Q}_{\mu}$. We have natural inclusions

$$
\operatorname{Rep}\left(Q_{\mu}, \alpha_{\mu}\right) \hookrightarrow \operatorname{Rep}\left(\widetilde{Q}_{\mu}, \alpha_{\mu}\right) \hookrightarrow \operatorname{Rep}(Q, \alpha)
$$

where the last inclusion is obtained by adding the vertex spaces of $Q_{\mu}$ (or $\tilde{Q}_{\mu}$ ) to obtain the vertex spaces for $Q$. A similar procedure applies to the matrices corresponding to arrows. Under this inclusion we have

Proposition 2.9. We can identify $S_{\mu}$ with $\operatorname{Rep}\left(\tilde{Q}_{\mu}, \alpha_{\mu}\right)$. Moreover, the action of $P(\lambda) \hookrightarrow G L(\alpha)$ on $S_{\mu}$ defines an action of $P(\lambda)$ on $\operatorname{Rep}\left(\tilde{Q}_{\mu}, \alpha_{\mu}\right)$ such that $L(\lambda)$ acts on the subspace $\operatorname{Rep}\left(Q_{\mu}, \alpha_{\mu}\right)$ by base change.

\section{UNISERIAL REPRESENTATIONS}

3.1. In this section we will apply the foregoing general results to the construction of moduli spaces for uniserial representations of the finite dimensional algebra $A$.

First, we will show that uniserial representations in $\operatorname{Rep}(Q, \alpha)$ can only belong to very special strata $S t_{\mu}$. We determine the structure of the quivers $Q_{\mu}$ and $\tilde{Q}_{\mu}$, and the dimension vector $\alpha_{\mu}$. In this case, the description of the $\theta_{\mu}$-semistable representations presents no problem.

In fact, we show that $T_{\mu}^{s s} / L(\lambda)$ is in this case a product of projective spaces. Over the standard open affine sets we can reduce the action of $P(\lambda)$ to that of the unipotent group $G=k_{+} \times \ldots \times k_{+}$(where the number of terms depends on the multiplicity with which the top-component occurs) on a slice which is $\operatorname{Rep}\left(\tilde{Q}_{\mu}^{s l}, \alpha_{\mu}\right)$ for a specific subquiver $\tilde{Q}_{\mu}^{s l}$ of $\tilde{Q}_{\mu}$. The orbits of this reduced action can be easily parameterized.

The results of Bongartz and Huisgen-Zimmermann in [1 can then be recovered using the equivariant embedding $\operatorname{Rep}(A, \alpha) \hookrightarrow \operatorname{Rep}(Q, \alpha)$.

The strategy of this classification extends to more general classes of representations. Let $\mu \in \operatorname{List}(Q, \alpha)$ be such that none of its segments contains numbers appearing with multiplicity $\geq 2$. Then, one can repeat almost verbatim the method below to classify $A$-representations having an optimal filtration corresponding to $\lambda$.

3.2. Fix a sequence $\mathbb{S}=(S(0), \ldots, S(l))$ of simple $A$-modules. Let $\alpha$ be the dimension vector $\left(a_{1}, \ldots, a_{n}\right)$, where $a_{i}$ is the number of times the simple $A$-module $S_{i}$ occurs among the $S(j)$. Observe that $l=a-1$, where, as before, $a=\sum_{i} a_{i}$.

We say that an $A$-module $M \in \operatorname{Rep}(A, \alpha)$ is filtered of type $\mathbb{S}$ if and only if $M$ has a decreasing Jordan-Hölder filtration

$$
M=M_{0} \supset M_{1} \supset \ldots \supset M_{l} \supset M_{l+1}=0
$$


with simple factors

$$
M_{i} / M_{i+1} \simeq S(i) \quad \forall i
$$

An $A$-module $M \in \operatorname{Rep}(A, \alpha)$ is said to be uniserial if and only if there is a unique (up to isomorphism) Jordan-Hölder filtration on $M$ with simple factors.

3.3. We have a $G L(\alpha)$-equivariant embedding

$$
\operatorname{Rep}(A, \alpha) \hookrightarrow \operatorname{Null}(Q, \alpha)=\bigsqcup_{\mu} G L(\alpha) . S t_{\mu}
$$

and we have determined which $\mu \in \operatorname{List}(Q, \alpha)$ actually occur.

By construction, $\tilde{Q}_{\mu}$ is a directed quiver. It is easy to determine all possible Jordan-Hölder filtrations on a representation of directed quivers.

If $W$ is a representation with dimension vector $\beta$ of the directed quiver $\Gamma$, then its socle $\operatorname{soc}(W)$ is the direct sum of the spaces at the sink-vertices of the subquiver of $\Gamma$ on the support of $\beta$ after removing the arrows for which the corresponding matrices of $W$ are zero.

Any (decreasing) Jordan-Hölder sequence on $W$ ends with one of the possible Jordan-Hölder filtrations on $\operatorname{soc}(W)$. Then, we repeat this procedure on the representation $\bar{W}=W / \operatorname{soc}(W)$.

Proposition 3.1. If $M \in \operatorname{Rep}(A, \alpha)$ is uniserial of type $\mathbb{S}=(S(0), \ldots, S(l))$, then up to A-module isomorphism $M$ belongs to $S_{\mu}$, where

$$
\mu=\sigma \cdot(q, q+1, q+2, \ldots, q+l)
$$

Here, $q=-\frac{l}{2}$ and $\sigma$ is the permutation of $S_{n}$ such that $\sigma(k) \in I_{v}$ if $S(k)=S_{v}$ and making $\mu$ dominant for the action of $S_{\alpha}$.

Proof. Up to $A$-isomorphism we may assume that $M \in S t_{\mu} \hookrightarrow S_{\mu}=\operatorname{Rep}\left(\tilde{Q}_{\mu}, \alpha_{\mu}\right)$. Clearly, if $M$ is uniserial, so is the corresponding representation $V$ of the directed quiver $\tilde{Q}_{\mu}$.

This means that at each step in the procedure to construct the Jordan-Hölder sequence on $V$ the socle must be one-dimensional.

In particular, all components of the dimension vector $\alpha_{\mu}$ must be equal to one or zero, and for each segment $J_{i}$ of $\mu$ and all $0 \leq j \leq k_{i}$ precisely one of the components of the vertices $(v, j)^{(i)}$ is equal to one, the others zero.

We claim that $\mu$ has only one segment. If not, there are vertices $v=\left(v_{1}, j_{1}\right)^{\left(i_{1}\right)}$ and $v^{\prime}=\left(v_{2}, j_{2}\right)^{\left(i_{2}\right)}$ with

$$
0<\left|p_{i_{2}}+j_{2}-p_{i_{1}}-p_{1}\right|<1
$$

and so there are no arrows in $\tilde{Q}_{\mu}$ between $v$ and $v^{\prime}$. But then, at some stage in the construction of the Jordan-Hölder sequence the dimension of the relevant socle is $\geq 2$, contradicting uniseriality.

Hence, writing the components of $\mu$ in increasing order, we get

$$
(q, q+1, \ldots, q+l) \text {. }
$$

As $\mu$ is balanced, its sum $(l+1) q+\frac{1}{2} l(l+1)=0$, finishing the proof. 
3.4. The full subquiver of $Q_{\mu}$ on the support of $\alpha_{\mu}$ is of the following form:

$$
Q_{\mu} \mid \operatorname{supp}\left(\alpha_{\mu}\right) \quad: \quad \stackrel{\left.a^{\prime}\right)}{a_{o 1}} \underset{(1)}{\longrightarrow} \underset{(2)}{a_{12}} \stackrel{a_{23}}{\longrightarrow} \cdots \quad \underset{(3)}{\stackrel{a_{l-1, l}}{(i)}}
$$

where the number $a_{i, i+1}$ of arrows from $(i)$ to $(i+1)$ is determined as follows. In the foregoing proof we have seen that there is a unique vertex $(i)$ among the vertices $(v, i)^{(1)}$ with dimension component equal to one. Let $V_{v}$ be the set of all vertices (i) in $Q_{\mu} \mid \operatorname{supp}\left(\alpha_{\mu}\right)$ corresponding to the vertex $v$ in $Q$. Observe that if $v=v_{j}$, then the number of elements of $V_{v}$ is equal to $a_{j}$. If $(i) \in V_{v}$ and $(i+1) \in V_{v^{\prime}}$, then $a_{i, i+1}$ is the number of arrows in $Q$ from $v$ to $v^{\prime}$.

From now on we will write $Q_{\mu}$ for $Q_{\mu} \mid \operatorname{supp}\left(\alpha_{\mu}\right)$ and $\alpha_{\mu}=(1, \ldots, 1)$ for $\alpha_{\mu} \mid \operatorname{supp}\left(\alpha_{\mu}\right)$.

If $l+1=a$ is odd we have $\lambda=\mu=\theta_{\mu}=(-k,-k+1, \ldots, k)$ with $l=2 k$. If $l+1=a$ is even, then $\lambda=\theta_{\mu}=(-l,-l+2, \ldots, l)$.

The Levi and parabolic subgroups of $G L(\alpha)$ are

- $L(\lambda)=T_{a} \hookrightarrow G L(\alpha)$,

- $P(\lambda)=B_{a_{1}} \times \ldots \times B_{a_{n}} \hookrightarrow G L(\alpha)$,

where $B_{a_{i}}$ is the Borel subgroup of lower triangular matrices in $G L_{a_{i}}$.

As $T_{\mu}=\operatorname{Rep}\left(Q_{\mu}, \alpha_{\mu}\right)$ and $\alpha_{\mu}=(1, \ldots, 1)$, it is easy to verify that the subvariety $T_{\mu}^{s s}$ of $\theta_{\mu}$-semistable representations consists of those representations of $\operatorname{Rep}\left(Q_{\mu}, \alpha_{\mu}\right)$ having no subrepresentation of dimension vector

$$
(1, \ldots, 1,0, \ldots, 0)
$$

These representations are precisely those for which at least one of the $a_{i, i+1}$ maps from $(i)$ to $(i+1)$ is non-zero for every $0 \leq i<l$. Observe that these representations are then uniserial, and under the canonical embedding

$$
\operatorname{Rep}\left(Q_{\mu}, \alpha_{\mu}\right) \hookrightarrow \operatorname{Rep}(Q, \alpha)
$$

they correspond to uniserial $Q$-representations of type $\mathbb{S}$.

In particular, $S t_{\mu} \neq \emptyset$ if and only if there is a path of length $l$ in the quiver $Q$ along the vertices $w_{0}, w_{1}, \ldots, w_{l}$ where $(i) \in V_{w_{i}}$.

Consequently, $A$ can only have uniserial modules in $\operatorname{Rep}(A, \alpha)$ of type $\mathbb{S}$ if at least one of these paths of length $l$ does not belong to the defining ideal of $A$. Compare this non-vanishing path with the notion of a 'mast' in [3, 4, 1].

3.5. With conventions as for $Q_{\mu}$, the extended quiver $\tilde{Q}_{\mu}$ is of the following form:

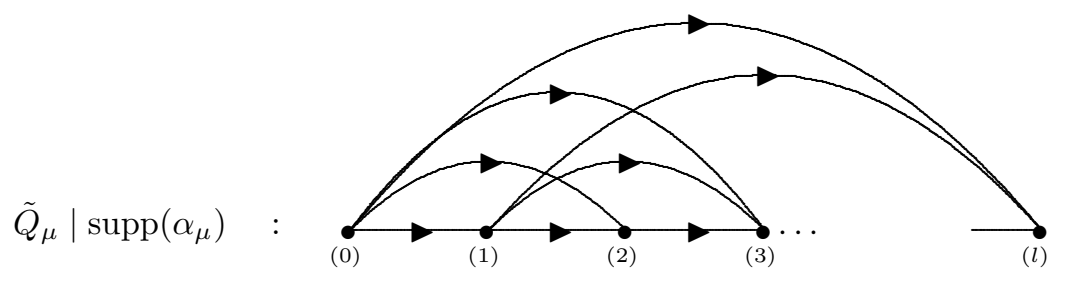

where the number of arrows $a_{i j}$ from $(i)$ to $(j)$ with $j>i$ is equal to the number of arrows in $Q$ from $v$ to $v^{\prime}$ if $(i) \in V_{v}$ and $(j) \in V_{v^{\prime}}$. 
As we have an explicit description of the open subset $T_{\mu}^{s s} \hookrightarrow T_{\mu}$, we can also describe the Hesselink stratum $S t_{\mu}$ explicitly as $p r^{-1}\left(T_{\mu}^{s s}\right)$, where

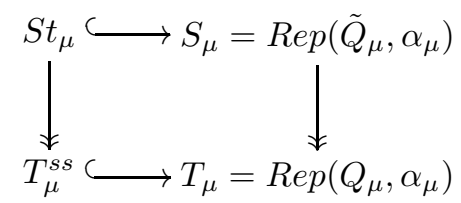

the fibers of $p r$ over vectorspaces of dimension $\sum_{j>i+1} a_{i j}$. Clearly, all representations of $S t_{\mu}$ are uniserial and correspond under the canonical inclusion

$$
\operatorname{Rep}\left(\tilde{Q}_{\mu}, \alpha_{\mu}\right) \hookrightarrow \operatorname{Rep}(Q, \alpha)
$$

to uniserial $Q$-representations of type $\mathbb{S}$.

3.6. We will now parameterize the isomorphism classes of uniserial representations of $\operatorname{Rep}(Q, \alpha)$ of type $\mathbb{S}$. Combining the foregoing remarks with the general results on Hesselink strata, this problem amounts to parameterizing the $P(\lambda)=B(\alpha)=$ $B_{a_{1}} \times \ldots \times B_{a_{n}}$-orbits in $S t_{\mu}$.

Recall that $T_{\mu}^{s s}$ is the set of representations in $\operatorname{Rep}\left(Q_{\mu},(1, \ldots, 1)\right)$ such that for every $0 \leq i<l$ at least one of the $a_{i, i+1}$ numbers determined by the arrows from (i) to $(i+1)$ is non-zero. As $L(\lambda)=T_{a}$ acting via

$$
\begin{aligned}
& \left(t_{0}, \ldots, t_{l}\right) \cdot\left(\left(m_{01}, \ldots, m_{0 a_{01}}\right), \ldots,\left(m_{l-11}, \ldots, m_{l-1 a_{l-1 l}}\right)\right) \\
= & \left(t_{1}^{-1} t_{0}\left(m_{01}, \ldots, m_{0 a_{01}}\right), \ldots, t_{l}^{-1} t_{l-1}\left(m_{l-11}, \ldots, m_{l-1 a_{l-1 l}}\right)\right)
\end{aligned}
$$

we observe that there is a geometric quotient

$$
T_{\mu}^{s s} \stackrel{b}{\rightarrow} T_{\mu}^{s s} / L(\lambda) \simeq \mathbb{P}^{a_{01}-1} \times \ldots \times \mathbb{P}^{a_{l-1, l}-1}
$$

We want to study the $P(\lambda)=B(\alpha)$-orbits in the open subvariety $U\left(i_{1}, \ldots, i_{l}\right)$ of $S_{\mu}=\operatorname{Rep}\left(\tilde{Q}_{\mu},(1, \ldots, 1)\right)$, which is the inverse image under the quotient map $b$ of the standard affine open subvariety

$$
\mathbb{A}^{a_{01}-1}\left(i_{1}\right) \times \ldots \times \mathbb{A}^{a_{l-1 l}-1}\left(i_{l}\right) \hookrightarrow \mathbb{P}^{a_{01}-1} \times \ldots \times \mathbb{P}^{a_{l-1, l}-1}
$$

that is, of points such that the $i_{j}$-th coordinate component of $\mathbb{P}^{a_{j-1, j}-1}$ is set equal to one.

Equivalently, $U\left(i_{1}, \ldots, i_{l}\right)$ can be identified with $\operatorname{Rep}\left(\tilde{Q}_{\mu}^{\mathbf{i}},(1, \ldots, 1)\right)$, where the quiver $\tilde{Q}_{\mu}^{\mathbf{i}}$ is the subquiver of $\tilde{Q}_{\mu}$ obtained by deleting these fixed arrows.

With this choice, the induced action of $T_{a}$ on $U\left(i_{1}, \ldots, i_{l}\right)$ is trivial and the action of $B(\alpha)$ reduces to an action of the nilpotent group

$$
U(\alpha)=U_{a_{1}} \times \ldots \times U_{a_{n}} \hookrightarrow G L(\alpha)
$$

where $U_{z}$ is the nilpotent radical of the Borel subgroup $B_{z}$ of $G L_{z}$.

3.7. In order to simplify the action of $U(\alpha)$ on $U\left(i_{1}, \ldots, i_{l}\right)$ even further, we have to describe the embedding

$$
\operatorname{Rep}\left(\tilde{Q}_{\mu},(1, \ldots, 1)\right) \hookrightarrow \operatorname{Rep}(Q, \alpha)
$$

For every $0 \leq i \leq l$ there exists a unique vertex $v(i)$ of $Q$ such that $(i) \in V_{v(i)}$. Let $j(i)=\#\left\{(k) \in V_{v(i)} \mid k \leq i\right\}$; then we will fix a basis of the vertex space of $Q$ in $v(i)$ by taking as its $j(i)$-th basevector a fixed vector spanning the one-dimensional vertex space of $\tilde{Q}_{\mu}$ in $(i)$. 
Take a representation $W \in \operatorname{Rep}\left(\tilde{Q}_{\mu},(1, \ldots, 1)\right)$ and consider an arrow $\gamma:(i) \rightarrow$ $(k)$ in $\tilde{Q}_{\mu}$. By definition $\gamma$ determines a unique arrow $\phi: v \rightarrow v^{\prime}$ in $Q$, where $(i) \in V_{v}$ and $(k) \in V_{v^{\prime}}$. By our assumption on the bases of the vertex spaces of $Q$ and $\tilde{Q}_{\mu}$ we have that

$$
\gamma=\phi_{j(k), j(i)}, \quad \text { where } \phi \in M_{\alpha_{k} \times \alpha_{i}}(k)
$$

We will construct a slice of the form $\operatorname{Rep}\left(\tilde{Q}_{\mu}^{s l},(1, \ldots, 1)\right)$, where $\tilde{Q}_{\mu}^{s l}$ is a subquiver of $\tilde{Q}_{\mu}^{\mathbf{i}}$ (and $\tilde{Q}_{\mu}$ ).

For every $(i)$ with $i \geq 1$, let $\phi_{i}$ be the arrow in $Q$ determined by the fixed arrow from $(i-1)$ to $(i)$ (the value of which we have set equal to 1 ). By the induced basechange action in $\operatorname{Rep}(Q, \alpha)$ by the subgroup $U(\alpha)$ we can ensure that the $j(i)$ th column of the matrix corresponding to $\phi_{i}$ contains this 1 as its only non-zero entry.

Performing the necessary computations, we see that this choice determines all components of the basechange matrix $\left(u_{1}, \ldots, u_{n}\right) \in U(\alpha)$ apart from the first column of $u_{v(0)}$. For, inductively we can determine the $j(1)$-th column of $u_{v(1)}$, then the $j(2)$-column of $u_{v(2)}$, and so on.

Consider the subquiver $\tilde{Q}_{\mu}^{s l}$ of $\tilde{Q}_{\mu}$ where we remove

- the fixed arrows from $(k)$ to $(k+1)$ determined by $i_{k}$ for every $0 \leq k<l$, and

- all arrows from $(k)$ to $(l)$ for $l>k$ corresponding to $\phi_{k}$.

By the argument given above we see that every $U(\alpha)$-orbit in $U\left(i_{1}, \ldots, i_{l}\right)$ contains a representation from $\operatorname{Rep}\left(\tilde{Q}_{\mu}^{s l},(1, \ldots, 1)\right)$.

The subgroup $G$ of $U(\alpha)$ preserving these indicated entries in $\operatorname{Rep}\left(\widetilde{Q}_{\mu},(1, \ldots, 1)\right)$ is a nilpotent subgroup of dimension $a_{v(0)}-1$ where $a_{v(0)}$ is the multiplicity with which the top component occurs in the Jordan-Hölder composition. Observe that the $G$ is not constant along $\operatorname{Rep}\left(\widetilde{Q}_{\mu},(1, \ldots, 1)\right)$ which forces us to decompose this space along pieces where $G$ is constant. I thank K. Bongartz and B. HuisgenZimmermann for pointing out this error in a prior version. For more details on this issue the reader is referred to 1 , in particular to their example 1.

We recall from [8, III.1.1,Satz 4] that all $G$-orbits in $\operatorname{Rep}\left(\tilde{Q}_{\mu}^{s l},(1, \ldots, 1)\right)$ are closed. Therefore, we can parameterize the orbits by stratifying according to the dimension of the stabilizer subgroup.

Each of the representing spaces can be identified to some $\operatorname{Rep}\left(Q^{\prime},(1, \ldots, 1)\right)$, where $Q^{\prime}$ is a subquiver of $\tilde{Q}_{\mu}^{s l}$, and for the corresponding stratum

$$
U_{Q^{\prime}} \hookrightarrow \operatorname{Rep}\left(\tilde{Q}_{\mu}^{s l},(1, \ldots, 1)\right)
$$

the quotient map

$$
U_{Q^{\prime}} \rightarrow \operatorname{Rep}\left(Q^{\prime},(1, \ldots, 1)\right)
$$

is a vectorbundle because all fibers are isomorphic to $\mathbb{A}^{s}$ for some $s \leq a_{v(0)}-1$.

3.8. Finally, using the equivariant embedding $\operatorname{Rep}(A, \alpha) \hookrightarrow \operatorname{Rep}(Q, \alpha)$ and our solution of the classification problem for the uniserial representations of $Q$ of type $\mathbb{S}$, we recover [1, Thm. A].

We mentioned already that the same construction can be repeated almost verbatim for representations having optimal filtrations corresponding to $\mu$ when none of the segments of $\mu$ contains numbers with multiplicity $\geq 2$. 
To begin the general classification problem of $A$-representations according to optimal filtration series, a natural idea would be to study the moduli spaces $T_{\mu}^{s s} / L(\lambda)$ studied by A. King in 6]. Over a suitable affine open cover of these projective varieties one might then try to construct slices reducing the acting parabolic group $P(\lambda)$ to a more manageable group.

\section{REFERENCES}

[1] K. Bongartz and B. Huisgen-Zimmermann, Varieties of uniserial representations IV : Kinship to geometric quotients, preprint UCSB (1997)

[2] W. Hesselink, Desingularization of varieties of nullforms, Invent. Math. 55 (1977), 141163 MR 81b:14025

[3] B. Huisgen-Zimmermann, The geometry of uniserial representations of finite dimensional algebras I, J. Pure Appl. Algebra 127 (1998), 39-72 MR 99b:16020

[4] B. Huisgen-Zimmermann, The geometry of uniserial representations of finite dimensional algebras III : Finite uniserial type, Trans. Amer. Math. Soc. 348 (1996) 4775-4812 MR 97f:16027

[5] G. Kempf, Instability in invariant theory, Ann. of Math. 108 (1978), 299-316 MR 80c:20057

[6] A. King, Moduli of representations of finite dimensional algebras, Quart. J. Math. Oxford 45 (1994) 515-530 MR 96a:16009

[7] F.C. Kirwan, Cohomology of quotients in symplectic and algebraic geometry, Princeton Math. Notes 31 (1984) MR 86i:58050

[8] H. Kraft, Geometrische Methoden in der Invariantentheorie, Aspects of Mathematics D1, Vieweg (1984) MR 86j:14006

[9] H. Kraft, Geometric methods in invariant theory, Representations of Algebras, Eds. M. Auslander and E. Lluis, Springer LNM 944 (1982) 180-258 MR 84c:14007

[10] L. Le Bruyn, Nilpotent representations, J. Alg. 197 (1997), 153-177 MR 98k:14070

[11] A. Schofield, General representations of quivers, Proc. LMS 65 (1992) 46-64 MR 93d:16014

[12] P. Slodowy, Die Theorie der optimalen Einparametergruppen für instabile Vektoren, DMV Seminar 13 (1989) Birkhäuser-Verlag, pp. 115-131 MR 91m:14074

Departement Wiskunde UiA Universiteitsplein 1, B-2610 Antwerp, Belgium

E-mail address: lebruyn@uia.ua.ac.be 